\title{
High-exitation nebulae around Magellanic Wolf-Rayet stars
}

\author{
Manfred W. Pakull ${ }^{1}$ \\ ${ }^{1}$ CNRS, Observatoire Astronomique 11, rue de l'Université, F67000 Strasbourg, France \\ email: pakull@astro.u-strasbg.fr
}

\begin{abstract}
The SMC harbours a class of hot nitrogen-sequence Wolf-Rayet stars (WNE) that display only relatively weak broad He II $\lambda 4686$ emission indicative of their low mass-loss rates and which are therefore hard to detect. However, such stars are possible emitters of strong $\mathrm{He}^{+}$Lyman continua which in turn could ionize observable He III regions, i.e. highly excited $\mathrm{H}$ II regions emitting nebular He II $\lambda 4686$ emission. We here report the discovery of a second He III region in the SMC within OB association NGC 249 within which the weak-lined WN star SMC WR10 is embedded. SMC WR10 is of special importance since it is a single star showing the presence of atmospheric hydrogen. While analysing the spectrum in the framework of two popular, independent WR atmosphere models we found strongly discrepant predictions (by 1 dex) of the $\mathrm{He}^{+}$continuum for the same input parameters. A second interesting aspect of the work reported here concerns the beautiful MCELS images which clearly reveal a class of strongly O III $\lambda 5007$ emitting (blue-coded) nebulae. Not unexpectedly, most of the "blue" nebulae are known Wolf-Rayet bubbles, but new bubbles around a few WRs are also detected. Moreover, we report the existence of blue nebulae without associated known WRs and discuss the possibility that they reveal weak-wind WR stars with very faint stellar He II $\lambda 4686$ emission. Alternatively, such nebulae might hint at the hitherto missing population of relatively low-mass, hot He stars predicted by massive binary evolution calculations. Such a binary system is probably responsible for the ionization of the unique He II $\lambda 4686$-emitting nebula $\mathrm{N} 44 \mathrm{C}$.
\end{abstract}

Keywords. shock waves, stars: winds, outflows, stars: Wolf-Rayet, ISM: bubbles, HII regions, Magellanic Clouds

\section{Wolf-Rayet stars and their $\mathrm{He}^{+}$ionizing radiation}

My interest for high-excitation H II regions dates back to the late 1980s. At that time I tried to understand the formation of the extended He II $\lambda 4686$ nebular recombination line in the HeIII region around the massive black-hole candidate LMCX-1 (Pakull \& Angebault 1986) in terms of photoionization and to draw conclusions about the otherwise unobservable EUV/soft X-ray ionizing continuum. X-ray ionization has been a well-understood process, but hitherto mainly applied to power-law ionizing continua in AGN. It turned out that the only known examples of nebular $\lambda 4686$ emission in $\mathrm{HII}$ regions concerned two Wolf-Rayet ring nebulae, one around the presumably very hot Galactic WO star WR102 and the other one surrounding a similar object in the Local Group galaxy IC 1613 (see, e.g., Pakull \& Motch 1989b and references therein). Another prominent He III region is located in the LMC, namely the small region N44C (= NGC 1936) within the larger H II complex N44. Here, the ionizing star is not a WR, but appears to be a normal O7 star. This led Pakull \& Motch (1989a) to propose an interpretation in terms of a fossil X-ray ionized nebula, possibly due to the transient source LMC X-5. As a historical remark, I'd like to mention that the presence of highly ionized He III regions around some WO stars led Terlevich \& Melnick (1985) to suggest that a 
population of very hot $\left(T_{\mathrm{rad}}>80-100 \mathrm{kK}\right)$ luminous stars might be responsible for the high ionization observed in AGN, thus challenging the general understanding in terms of massive accreting black holes. For such stars they coined the fitting term Warmers.

In 1991, at the Bali IAU Symposium 143 on Wolf-Rayet stars two groups (Niemela et al. 1991; Pakull 1991; Pakull \& Bianchi 1991) independently announced the discovery of He III regions around a few Magellanic WR stars. They were SMC WR7 (located in $\mathrm{N} 76$ ) in the SMC, and in the LMC Brey 2 (located in N79) and Brey 40a (within an anonymous H II region). The spectral types of these Magellanic "Wolf-Rayet Warmers" (hereafter WRW) came as a surprise: these stars are early WN types rather than the more advanced WO types as one might have naively expected from the previously recognized objects. Moreover, it was shown that the two known Magellanic WOs do not excite observable He III regions (Pakull 1991), i.e., a WO is not necessarily a WRW! Since then, and although several additional Magellanic WR stars have been discovered in the meantime, no new WRW turned up in the MCs.

Since the pioneering work by Schmutz, Leitherer \& Gruenwald (1992) we know that the ionizing radiation from WR stars does not only depend on the atmospheric "core" temperature of the star (i.e., at a radius where the wind is still subsonic), but also on the mass-loss rate. In short, a strong wind from a WN star (consisting mainly of helium) will absorb all $\mathrm{He}^{+}$Lyman continuum photons (i.e., $h \nu>54 \mathrm{eV}$ ), not unlike an ionizationbound Strömgren sphere. If however the wind is sufficiently weak, a large fraction of the hard ionizing photons will escape and create observable He III regions in the surrounding ISM. Grids of the most recent Wolf-Rayet star models which among others also take into account line-blankening are described in Smith et al. (2002) (CMFGEN models) and in Hamann \& Gräfener (2004) (PoWR models). Note that the CMFGEN grid is implemented in the popular Starburst99 software package. As will be shown below, and contrary to what is often claimed or assumed, the two models strongly disagree on the predicted power of the emitted $\mathrm{He}^{+}$Lyman continuum emission.

The study of Wolf-Rayet (WR) stars currently witnesses a renaissance since it was realised that these stars - or rather a not-yet-specified rare subclass thereof - appear to be the direct progenitors of (long) gamma ray bursts (cf., Yoon \& Langer 2005). One important ingredient of currently favoured models is very high rotation of the core at the time of collapse/jet formation which however is not easily realized due to expected angular momentum loss suffered during their evolution due to stellar wind.

At low metallicity, WR winds are now known (cf., Vink \& de Koter 2005 and references therein) to be weaker than those of their more metal-rich Galactic counterparts, and it is possible that under these circumstances (low $Z$, very rapid rotation) massive stellar evolution proceeds quasi-chemically homogeneously (Yoon \& Langer 2005). In this scenario, the stars remain in the blue part of the HR diagram and evolve from the main sequence directly "bluewards" to high effective temperatures $\left(T_{\text {eff }}>70-100 \mathrm{kK}\right)$ even if their outer layers still contain hydrogen. It is however an open question whether homogeneous evolution is indeed realized in nature.

\section{SMC WR10: A second Warmer in the Small Cloud}

Shortly after the somewhat unexpected discovery of several new WR stars in the SMC (Massey \& Duffy 2001) we obtained long-slit spectra of the newly identified WR10 which is located within the H II region NGC 249 (Fig. 1). Although this object has subsequently also been observed with various similar instrumental set-ups no other observer seems to have noticed (or have cared to look at) the relatively strong extended He II $\lambda 4686$ emission that is depicted in Fig. 2. 


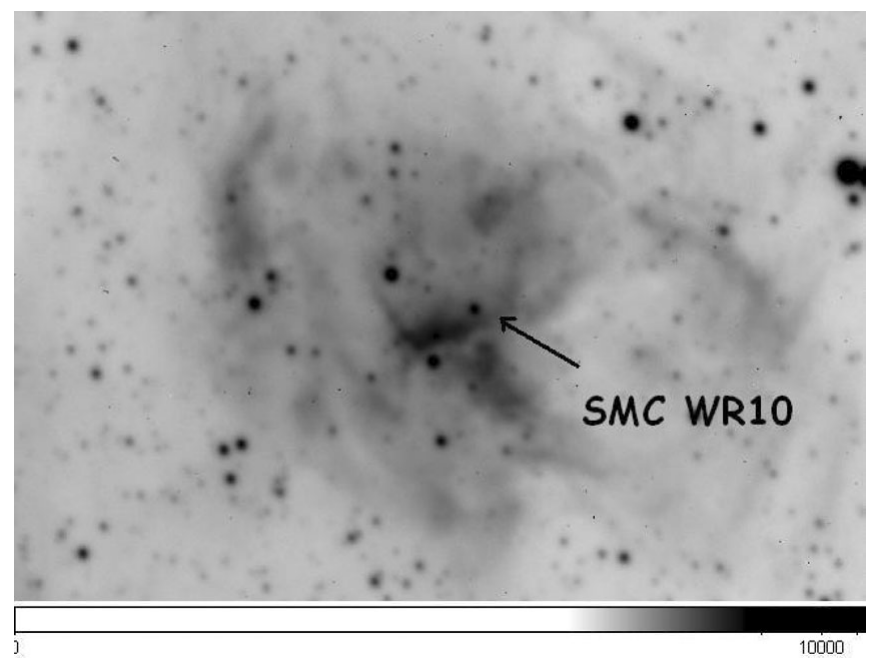

Figure 1. ESO NTT/EMMI H $\alpha$ image of the SMC H II region NGC 249 in which the Wolf-Rayet star SMC WR10 (WN3ha) is embedded. North is up and East is to the left. The image covers $3^{\prime} \times 2^{\prime}$.

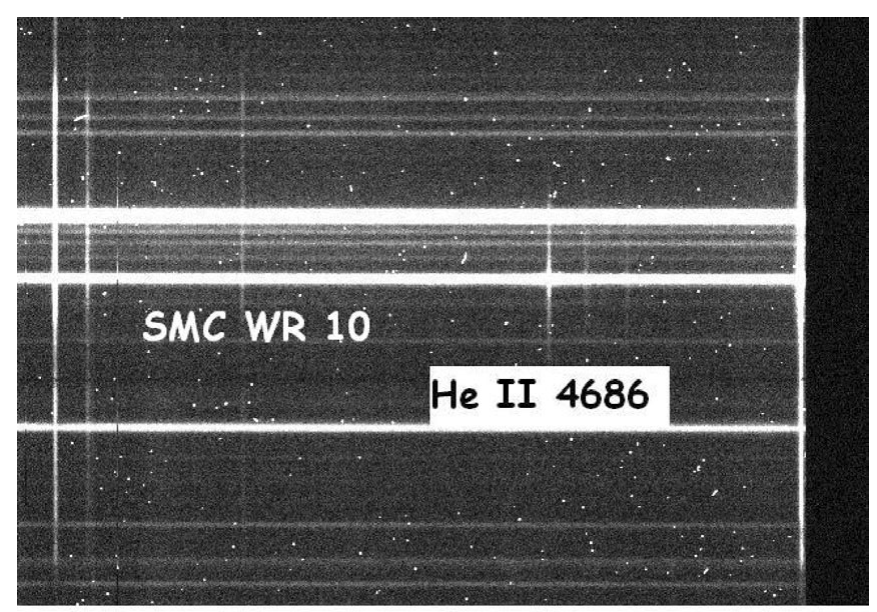

Figure 2. ESO NTT/EMMI Long-slit spectrum of SMC WR10 and the surrounding H II region NGC 249. The strong vertical lines to the left and to the right are nebular $\mathrm{H} \gamma$ and $\mathrm{H} \beta$, respectively. The He II $\lambda 4686$ emitting He III region extends $45^{\prime \prime}$ corresponding to some $10 \mathrm{pc}$ in the SMC.

The particular importance of SMCWR10 for our understanding of the physics and evolution of WR stars comes from the fact that this WN3ha star is most likely single (Foellmi, Moffat \& Guerro 2003) and therefore probably not a result of massive binary evolution. Moreover, the star is faint $(V \sim 16.0 \mathrm{mag})$, displays a rather weak $(E W=25$ $\AA$ ) and narrow ( $F W H M=30 \AA$ ) stellar He II $\lambda 4686$ emission line (Crowther \& Hadfield 2006), and it contains substantial amounts of hydrogen in its atmosphere.

An important diagnostic for WRWs is the flux ratio, $R$, between the narrow nebular and the broad stellar He II $\lambda 4686$ emission. $R$ directly measures the fraction of $\mathrm{He}^{+}$ionizing radiation that escapes the Wolf-Rayet wind. For SMC WR10 one finds $R \sim 5$ 
which is similar to the $R$ values found for WRWs Brey 2 and SMC WR7. Fitting this set of observations with the Potsdam WR model WNL grid (cf., Hamann \& Gräfener 2004) one readily derives the following stellar parameters:

$T_{\star}=100 \pm 10 \mathrm{kK}, R_{\star}=2.4 \pm 0.3 \mathrm{R}_{\odot}, L=10^{5.7 \pm 0.2} \mathrm{~L}_{\odot}, \dot{M}=210^{-6} \mathrm{M}_{\odot}$.

Note in particular the small derived mass-loss rate as compared to more typical WolfRayet winds that are 10 to 100 times more powerful. Interestingly, the Smith et al. (2002) CMFGEN models suggest a significantly smaller temperature $T_{\star}=80 \mathrm{kK}$ for the same $\mathrm{He}^{+} / \mathrm{H}^{0}$ Lyman continuum ratio. In other words, for the same core temperature, CMFGEN models predict roughly $10 \times$ more $\mathrm{He}^{+}$-ionizing photons than the corresponding PoWR models! On my request, Wolf-Rainer Hamann kindly computed weakwind PoWR models with exactly the same input parameters as the hot low metallicity $\left(0.2 \mathrm{Z}_{\odot}\right)$ stars listed in Smith et al. (2002). He found the same discrepancy and suggested that possibly different assumed gravities of the underlying cores might play a role here.

As far as I am aware no standard evolutionary track leads to objects like SMC WR10. However, as mentioned earlier, for very rapid rotation evolution of massive stars is expected (Yoon \& Langer 2005) to proceed directly towards high effective temperatures into the region of the Hertzsprung-Russell diagram where SMC WR10 is located. Quite possibly, this is the first time that quasi-chemically homogeneous evolution has been substantiated.

\section{Highly excited "blue" nebulae in the MCELS images}

Recently, Smith and the MCELS team (cf., Smith et al. 1999) published beautiful multi-colour images of the Magellanic Clouds emphasizing nebular emission of $\mathrm{H} \alpha$ (red color coded; $R$ ), O III $\lambda 5007$ (blue; $B$ ), and S II $\lambda 6716,31$ (green; $G$ ). These images are not only very appealing (which explains why they have been shown in many talks during the conference!), but they also allow to readily discriminate between various ionization/excitation mechanisms at work.

These images easily allow to identify green and yellow $(y=R+G)$ shock-ionized SNR, often superimposed on normal reddish $\mathrm{H}$ II regions, and contemplate the ionization structure of photoionized nebulae that often turn "yellowish" and "brownish" $(=y+R)$ towards the edges where recombination towards lower-ionization species occurs.

Even a casual inspection of the LMC image in particular readily reveals a class of very blue nebulae (hereafter "BNe"); i.e. nebulae that appear to be dominated by the O III $\lambda 5007$ blue hue of the image. I have verified that the colour balance is such that nebulae appear "very blue" when the intensity ratio O III $\lambda 5007 / \mathrm{H} \alpha>1.5$; i.e., being of very high excitation. I detect some $40 \mathrm{BNe}$ in the LMC (not including the 30 Dor region which appears "burned-out") and about 10 BNe in the SMC, excluding NGC 346 and environment (cf. Fig. 3.)

What is the nature of the BNe? By employing the Strasbourg Aladin SKY AtLAS one quickly realizes that a large fraction thereof represents photoionized nebulae around known early WN stars (WNE), several of which were not known before to excite ring nebulae around them (cf. the compilation by Dopita et al. (1994), and SMC WR update by Massey \& Duffy (2001)). Late WN (WNL) and WC types appear to create less highly excited (violet-red coloured) nebulae more akin to more normal O star H II regions. The fact that such subtle differences become so strikingly apparent is indeed quite remarkable. It is also clear that many WNE BNe are much more extended than previously thought, and several such bubbles have O III $\lambda 5007$ - "skin" emission due to photoionized/incomplete shocks in the supersonically expanding bubbles (see, e.g., Dufour 1989). 

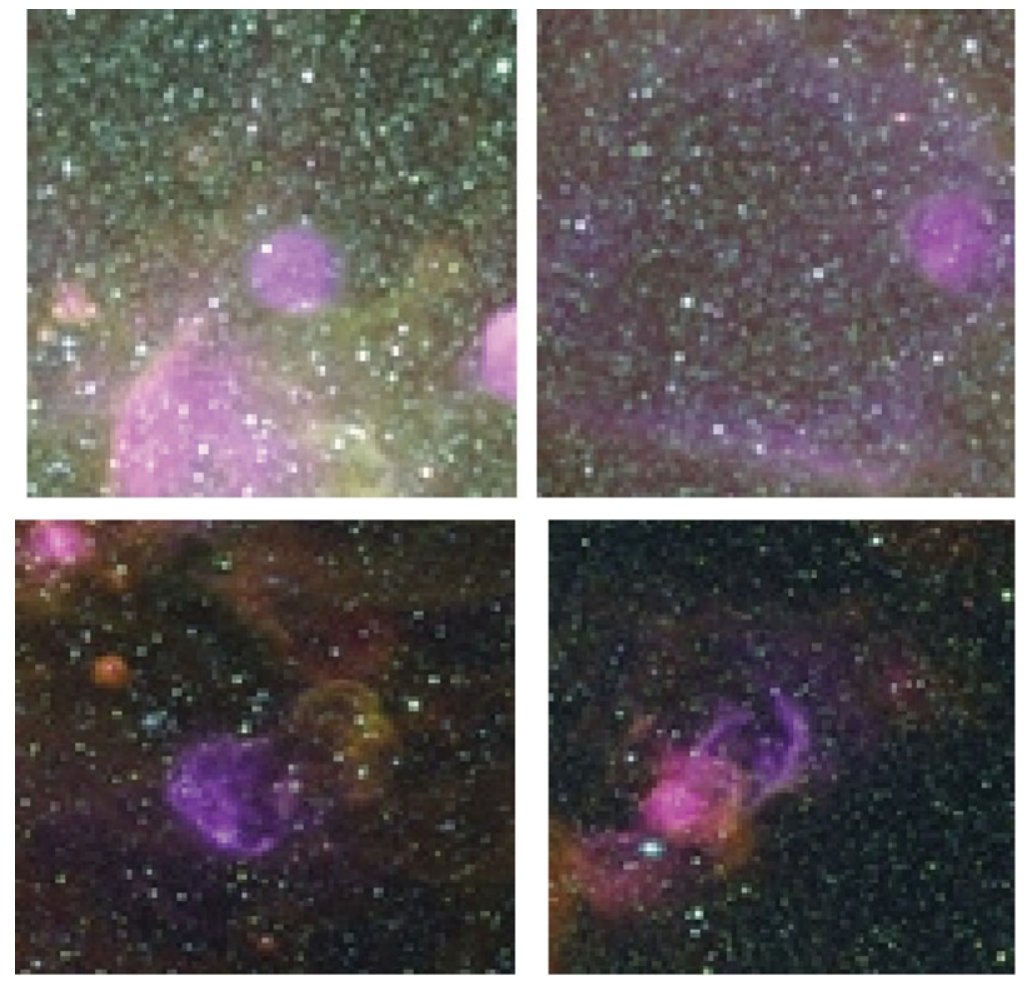

Figure 3. Examples of strongly O III $\lambda 5007$ emitting blue nebulae in the Magellanic Clouds. These cut-outs each measure about $10^{\prime} \times 10^{\prime}$ corresponding to $150 \mathrm{pc}$ in the Clouds. One needs to look at the colour version of the images in order to appreciate the unique "blueness" of the nebulae. From upper left, clockwise: (a) small round nebula North of the H II region N 19 (below) in the SMC, (b) previously unknown ring nebula around the faint WNE star SMC WR9, (c) a blue ring nebula in DEM $66(=\mathrm{N} 23 \mathrm{~A})$ in the LMC (d) the high excitation blue nebula NGC 1945 in the LMC located next to low exitation nebula NGC 1948 to the W (right)

We then might ask the question: What is the nature of the BNe not clearly associated with (known) WNEs? Remote possibilities include flash-ionized nebula around young Orich supernova remnants (like in the case of SNR 0103-72.6), rare X-ray ionized nebulae or regions of incomplete shocks at the periphery of more easily discernable low-excitation SNRs. However, a more likely possibility is that we see here the fingerprints of hidden WN stars that have but weak stellar emission lines, i.e., being even fainer than those of the recently identified class of weak-lined SMC WRs. Such stars could have easily escaped detection by using slitless spectroscopy or narrow filter imaging techniques. Also, such objects would have weak winds and therefore be prone to be WRW. A possible example is the huge (150 pc diameter) newly detected faint ring nebula around SMC WR9 (a spectroscopic twin of WR 10) shown in Fig. 3.

\section{A remark on the nature of He II $\lambda 4686$ nebula $\mathrm{N} 44 \mathrm{C}$}

Not unexpectedly, He III region N 44C also appears as a prominent BN, in the MCELS image of the Large Cloud. However, its ionizing source has not been clearly identified even though an interpretation in terms of a fossil X-ray ionized nebula cannot be excluded. Here I propose an alternative scenario, not least inspired by the variable radial 
velocity of the central O7 star (Pakull 1989) which clearly points to a binary nature. Indeed, evolutionary calculations of massive binaries by Wellstein, Langer \& Braun (2001) predict a sizable population of massive $\mathrm{O}$ stars with low-mass, $\left(5-7 \mathrm{M}_{\odot}\right)$, hot $\left(\sim 10^{5} \mathrm{~K}\right)$ Helium-star secondaries after mass transfer from the formerly more massive component to its companion has been completed. The He stars will be much fainter optically than the $\mathrm{O}$ star primaries and will therefore be quasi undetectable spectroscopically, readily explaining the apparent absence of such systems. However, the idea here is that such a star will act as a Warmer to excite a He III region that will in turn be detectable as a He II $\lambda 4686$ emitting nebula provided that the interstellar density is sufficiently high. Conceivably, the central (binary) star in N $44 \mathrm{C}$ is such a system.

\section{Acknowledgements}

I thank the editors for their patience with the delivery of my manuscript.

\section{References}

Crowther, P. A. \& Hadfield, L. J. 2006, A\& A, 449, 711

Dopita, M. A., Bell, J. F., Chu, Y. -H., \& Lozinskaya, T. A. 1994, ApJS, 93, 455

Dufour, R. J. 1989, Rev. Mexicana AyA, 18, 87

Foellmi, C., Moffat, A. F. J., \& Guerro, M. A. 2003, $M N R A S, 338,360$

Hamann, W.-R. \& Gräfener, G. $2004 A \mathscr{E} A$, 427, 697 — http://www.astro.physik.uni-potsdam. $\mathrm{de} / \sim \mathrm{wrh} / \mathrm{PoWR} /$ powrgrid1.html

Massey, P. \& Duffy, A. S. 2001, ApJ, 550, 713

Niemela, V. S., Heathcote, S. A., \& Weiller, W. C. 1991, in K.A. van der Hucht \& B. Hidayat (eds.), Wolf-Rayet stars and interrelations with other massive stars in galaxies, IAU Conf.Proc. 143 (Dordrecht: Kluver), p. 425

Pakull, M. W. \& Motch, C. 1989a, Nature 337, 337

Pakull, M. W. \& Motch, C. 1989b, in E. J. A. Meurs \& R. A. E. Fosbury (eds.), ESO workshop on extranuclear activity in galaxies (Garching: ESO), p. 285

Pakull, M. W. 1989, in K. S. de Boer, F. Spite, \& G. Stasińska (eds.), Recent developments in Magellanic Cloud research (Meudon: Observatoire de Paris), p. 183

Pakull, M. W. 1991, in K. A. van der Hucht \& B. Hidayat (eds.), Wolf-Rayet stars and interrelations with other massive stars in galaxies, IAU Conf.Proc. 143 (Dordrecht: Kluver), p. 391

Pakull, M. W. \& Bianchi, L. 1991, in K. A. van der Hucht \& B. Hidayat (eds.), Wolf-Rayet stars and interrelations with other massive stars in galaxies, IAU Conf.Proc. 143 (Dordrecht: Kluver), p. 260

Pakull, M. W \& Angebault, L. P. 1986, Nature, 322, 511

Schmutz, W., Leitherer, C., \& Gruenwald, R. 1992, PASP, 104, 1164

Smith, R. C. \& the MCELS Team 1999, in Y. -H. Chu, N. Suntzeff, J. Hesser, \& D. Bohlender (eds.), New views of the Magellanic Clouds, IAU Conf.Proc. 190, p. 28 http://www.ctio.noao.edu/ moels/

Smith, L. J., Norris, R. P. F., \& Crowther, P. A. 2002, MNRAS, 337, 1309

Terlevich, R. \& Melnick, J. 1985, MNRAS, 213, 841

Vink, J. S. \& de Koter, A. 2005, A\&A, 442, 587

Wellstein, S., Langer, N., \& Braun, H. 2001, A\&BA, 369, 939

Yoon, S. -C. \& Langer, N. 2005, A\&A A, 443, 643 